\title{
Morbidity Rate of the Retrosigmoid versus Translabyrinthine Approach for Vestibular Schwannoma Resection
}

\author{
Sami Obaid ${ }^{1}$, Ioannis Nikolaidis ${ }^{1}$, Musaed Alzahrani $^{2}$, Robert Moumdjian $^{1}$, and Issam Saliba ${ }^{2}$ \\ ${ }^{1}$ Divisions of Neurosurgery, ${ }^{2}$ Otolaryngology, Head and Neck Surgery, Department of Surgery, \\ Montreal University Hospital Center (CHUM), Montreal, Canada
}

\begin{abstract}
Received March 26, 2018
Revised May 22, 2018

Accepted June 21, 2018
\end{abstract}

\section{Address for correspondence Issam Saliba, MD, FRCSC \\ Division of Otolaryngology, \\ Head and Neck Surgery, \\ Department of Surgery, \\ Montreal University Hospital Center \\ (CHUM), 1051 Sanguinet St, \\ Montreal-QC, H2X 3E4, Canada \\ Tel +1-514-507-7722 \\ Fax +1-514-737-4822 \\ E-mail issam.saliba@umontreal.ca}

Background and Objectives: Controversy related to the choice of surgical approach for vestibular schwannoma (VS) resection remains. Whether the retrosigmoid (RS) or translabyrinthine (TL) approach should be performed is a matter of debate. In the context of a lack of clear evidence favoring one approach, we conducted a retrospective study to compare the morbidity rate of both surgical approaches. Subjects and Methods: 168 patients underwent surgical treatment (2007-2013) for VS at our tertiary care center. There were no exclusion criteria. Patients were separated into two groups according to the surgical approach: TL group and RS group. Signs and symptoms including ataxia, headache, tinnitus, vertigo and cranial nerve injuries were recorded pre- and postoperatively. Surgical complications were analyzed. Perioperative facial nerve function was measured according to House-Brackmann grading system. Results: Tumor resection was similar in both groups. Facial paresis was significantly greater in RS group patients preoperatively, in the immediate postoperative period and at one year follow-up $(p<0.05)$. A constant difference was found between both groups at al three periods $(p=0.016)$. The evolution of proportion was not found to be different between both groups $(p=0.942)$, revealing a similar rate of surgically related facial paresis. Higher rate of ataxic gait $(p=0.019)$, tinnitus $(p=0.039)$ and cranial nerve injuries $(p=0.016)$ was found in RS group patients. The incidence of headache, vertigo, vascular complications, cerebrospinal fluid leak and meningitis was similar in both groups. No reported mortality in this series. Conclusions: Both approaches seem similar in terms of resection efficacy. However, according to our analysis, the TL approach is less morbid. Thus, for VS in which hearing preservation is not considered, $\mathrm{TL}$ approach is preferable.

J Audiol Otol 2018;22(4):236-243

KEY WORDS: Vestibular schwannoma · Acoustic neuroma - Retrosigmoid · Translabyrinthine Complications $\cdot$ Morbidity.

\section{Introduction}

Observation, stereotactic radiotherapy (SR), surgical removal or combined approaches are all accepted treatment options for vestibular schwannomas (VS), with no clear established criteria for treatment selection. Due to their unpredictable evolution, observation with regular follow-up by magnetic resonance imaging (MRI) is an option in carefully selected patients $[1,2]$. Surgical management should be con-

This is an Open Access article distributed under the terms of the Creative Commons Attribution Non-Commercial License (https://creativecommons.org/licenses/by-nc/4.0/) which permits unrestricted non-commercial use, distribution, and reproduction in any medium, provided the original work is properly cited. sidered for large tumors in healthy patients [3-5], considering challenging adequate SR results for tumors of this size. It is also the recommended primary modality for the treatment of large, symptomatic or growing VSs [6,7].

Surgical approaches for resection of VSs have evolved considerably in the last few decades. Indeed, important contribution from various surgeons in the 20th century including Dandy [7] and House [8] has lead us to refining the different approaches performed nowadays. Although technically different, all surgical approaches aim to maximize total tumor removal with preservation of neurological functions [9-11].

Controversy related to the choice of surgical approach remains. Whether the retrosigmoid (RS) or translabyrinthine 
(TL) approach should be performed is a matter of debate. The TL approach is generally considered ideal in patients with non-serviceable hearing (Gardner-Robertson Class III or IV hearing level) since complete hearing loss will ensue following resection. The belief that this approach is associated with a lower rate of facial nerve paresis due to earlier identification of the facial nerve [12] remains controversial, with studies revealing a higher risk of facial injury than its counterpart $[9,13,14]$. On the other hand, even though the RS approach allows for hearing preservation in small tumors, its technical aspects seem to be associated with higher incidence of postoperative headache and cerebrospinal fluid (CSF) leak $[9,15]$. Additional postoperative complications including other cranial nerve palsies, cerebellar and vascular complications have been compared for both approaches [12-14,16,17]. Nevertheless, no reproducible conclusions have been identified. For that reason, we conducted a retrospective study to compare the morbidity rate of both surgical approaches in our institution.

\section{Subjects and Methods}

Between January 2007 and December 2013, 168 patients underwent surgical treatment for VS at our tertiary care center. All patients were selected for a retrospective chart review. Data collection was performed by one single individual. Preand postoperative clinical and radiological data were reviewed. The following information was recorded: age, sex, affected side, initial signs and symptoms, tumor surface, size in the internal auditory canal (IAC), extension anterior to the longitudinal axis of the IAC, extent of surgical removal (total versus partial) based on preoperative visualization and postoperative imaging, duration of surgery, hospital stay, duration of followup and surgical complications.

All patients that underwent surgical resection for VS through a TL or RS approach were included. There were no exclusion criteria. All tumor characteristics were measured on Siemens SOMATOM Sensation 16 or 64-slice CT scanners (Siemens Healthcare GmbH, Erlangen, Germany) with a slice thickness of $3 \mathrm{~mm}$. Estimated tumor surface on axial plane was obtained by multiplying the two largest extrameatal diameters using planimetric measurements. MRI was not available for all cases justifying the use of CT scan measurements.

Neurophysiological facial nerve monitoring was used for all cases. Facial nerve function both pre- and postoperatively was measured according to the House-Brackmann (HB) grading system [18].

Patients were divided in two groups. TL group represents patients who underwent VS resection through a TL approach, whereas RS group includes patients treated by RS approach.
Groups were divided depending on the main surgeon: when the main surgeon was a neurosurgeon assisted by the neurotologist, RS approach was chosen; when the main surgeon was a neurotologist assisted by the neurosurgeon, TL approach was chosen. The TL and RS technical aspects performed for patients in their respective groups have already been described in details previously [19].

Although surgeon's preference influenced the selection of one approach over the other, preservation of preoperative hearing status as well as the size of the tumor $(\leq 1.5 \mathrm{~cm})$ favored the RS approach. On the other hand, the TL approach was performed in cases of non-serviceable hearing and larger tumor sizes, considering the potential preservation of facial function often described with this technique.

All patients signed the informed consent form. This study was approved by our Institutional Review Board and got the ethics committee approval number: CE 08.099.

\section{Statistical analysis}

The Pearson chi-square test was used to compare postoperative data in both groups for the following categorical variables: extent of surgical removal, postoperative vascular complications, CSF leak and meningitis.

Student t-test analysis was used for comparison of continuous variables including age, estimated tumor surface, size in the IAC, extension in front of the IAC, duration of surgery, hospital stay and duration of follow-up.

Two-sided analysis via Pearson chi-square test of both preand postoperative vertigo, headache, ataxia, tinnitus and nonfacial cranial nerve injuries was performed: the number of cases that improved, deteriorated or remained stable were recorded in order to correlate any change in those parameters to the surgical procedure. Thus, data in both the pre- and postoperative period were necessary to analyze the evolution. Data for postoperative ataxia, vertigo, headache, and tinnitus was recorded one month following surgical intervention, while cranial nerve injuries were analyzed in the immediate postoperative course.

ANOVA of repeated measures was used for analysis of facial paresis preoperatively, in the immediate postoperative period and at one-year follow-up. Clinical cut-off for facial paresis was defined as $\mathrm{HB}$ of more than II. A $p$ value less than 0.05 was chosen to indicate statistical significance.

\section{Results}

\section{Patient population}

168 patients were included for analysis: 129 patients underwent TL VS resection while 39 underwent RS VS resec- 
tion. TL resection is a more frequently performed technique at our institution explaining the larger amount of patients included in that group. Despite this discrepancy, groups were statistically comparable.

\section{TL group}

129 patients were included in this group. Their mean age was 51.91 years (range 22-80 years) with a near equal malefemale and right-left side distribution (53\% female; $51 \%$ right side). None of these patients had undergone previous surgery or radiation therapy. The mean follow-up duration was 17 months (range $1-119$ months).

\section{RS group}

39 patients were included in this group. The mean age was 51.77 years (range $24-79$ years). Female predominance (54\%) and a higher rate of right-sided lesions (25 patients, 64.1\%) were also observed. None of these patients had undergone surgery or radiation therapy prior to admission. The mean follow-up duration was 45 months (range 3-118 months).

\section{Preoperative tumor characteristics}

Data was obtained for all 168 patients. Estimated tumor surface was comparable for both groups, revealing a mean of $4.64 \mathrm{~cm}^{2}$ for TL group and $3.66 \mathrm{~cm}^{2}$ for RS group ( $\left.p=0.325\right)$. Mean extension anterior to the longitudinal axis of the IAC was

Table 1. Preoperative tumor characteristics

\begin{tabular}{lcc}
\hline \multirow{2}{*}{ Tumor characteristics } & \multicolumn{2}{c}{ Approach } \\
\cline { 2 - 3 } & TL group & RS group \\
\hline Number of patients & 129 & 39 \\
Mean estimated surface $\left(\mathrm{cm}^{2}\right)$ & 4.64 & 3.66 \\
Extension anterior to the IAC $(\mathrm{cm})$ & 0.11 & 0.67 \\
Size in the IAC $(\mathrm{cm})$ & 0.80 & 0.94
\end{tabular}

p>0.05 according to student t-test for all characteristics. TL: translabyrinthine, RS: retrosigmoid, IAC: internal auditory canal measured at $0.11 \mathrm{~cm}$ for TL group and $0.67 \mathrm{~cm}$ for RS group ( $p=0.192)$, thus revealing no statistically significant difference. Comparison of the intracanalar extension between both groups $(0.80 \mathrm{~cm}$ and $0.94 \mathrm{~cm}$ for TL group and RS group, respectively) showed no difference $(p=0.615)$ (Table 1$)$. Hence, tumor characteristics were considered comparable for both groups.

\section{Initial presentation}

At initial presentation, the rate of patients presenting with ataxic gait, headache, vertigo and non-facial cranial nerve injuries was higher in RS group. Statistically significant difference was revealed for ataxia and cranial nerve injuries. On the other hand, tinnitus was significantly more frequent in TL group patients (Table 2). Finally, facial nerve function was analyzed: no patients of TL group vs. 6 patients of RS group $(15.8 \%)$ exhibited facial paresis preoperatively.

\section{Intraoperative parameters}

Total resection was similar in both groups (TL group: 85\%; RS group: $72 \%, p=0.079)$. Difference in the mean duration of surgery between both groups was found to be significantly different (TL group: 6.43 hrs; RS group: 8.19 hrs, $p=0.004$ ).

\section{Postoperative events}

\section{Facial paresis}

In the immediate postoperative period, facial paresis was noted in 37 patients of TL group (28.7\%) and 16 patients of RS group (41\%). At one-year follow-up, the HB of 14 cases $(12.7 \%)$ and 9 cases (28\%) of TL and RS group respectively was greater than II. In their respective group, a significant increase in facial paresis was found between the preoperative and the immediate postoperative period ( $p<0.001)$; an important improvement was observed between the immediate

Table 2. Evolution of signs and symptoms following surgical excision

\begin{tabular}{|c|c|c|c|c|c|c|c|c|c|c|}
\hline & \multicolumn{10}{|c|}{ Approach } \\
\hline & \multicolumn{5}{|c|}{ TL group } & \multicolumn{5}{|c|}{ RS group } \\
\hline & $\begin{array}{c}\text { Initial } \\
\text { presentation (\%) }\end{array}$ & $\begin{array}{l}\text { Available } \\
\text { cases }^{\dagger}\end{array}$ & $\begin{array}{c}\text { Worsened } \\
(\%)\end{array}$ & $\begin{array}{l}\text { Stable } \\
(\%)\end{array}$ & $\begin{array}{c}\text { Improved } \\
(\%)\end{array}$ & $\begin{array}{l}\text { Initial } \\
\text { presentation (\%) }\end{array}$ & $\begin{array}{l}\text { Available } \\
\text { cases }^{*}\end{array}$ & $\begin{array}{c}\text { Worsened } \\
\text { (\%) }\end{array}$ & $\begin{array}{c}\text { Stable I } \\
(\%)\end{array}$ & $\begin{array}{c}\text { Improved } \\
(\%)\end{array}$ \\
\hline Ataxic gait* & $41 / 112(36.6)^{\dagger}$ & 96 & $11(11.5)$ & $61(63.5)$ & $24(25)$ & $20 / 39(51.3)$ & 25 & $7(28)$ & $17(68)$ & $1(4)$ \\
\hline Headache & $27 / 112(24.1)^{\dagger}$ & 95 & $6(6.5)$ & $74(77.9)$ & $15(15.8)$ & 18/39 (46.2) & 22 & $3(13.6)$ & $15(68.2)$ & $4(18.2)$ \\
\hline Tinnitus* & $92 / 114(80.7)^{\dagger}$ & 106 & $2(1.9)$ & $37(34.9)$ & $67(63.2)$ & $22 / 38(57.9)^{\dagger}$ & 36 & $1(2.8)$ & $21(58.3)$ & $14(38.9)$ \\
\hline Vertigo & $46 / 112(41.1)^{\dagger}$ & 107 & $12(11.2)$ & $71(66.4)$ & $24(22.4)$ & $24 / 39(61.5)$ & 36 & $3(8.3)$ & $25(69.4)$ & $8(22.2)$ \\
\hline CN injuries* & $7 / 129(7.4)$ & 129 & $4(3.1)$ & $124(96.1)$ & $1(0.8)$ & $7 / 39$ (17.9) & 39 & $6(15.4)$ & $33(84.6)$ & $0(0)$ \\
\hline
\end{tabular}

Worsening, stability and improvement are obtained by comparing the postoperative to the preoperative signs and symptoms. Postoperative data was obtained at one month with the exception of $\mathrm{CN}$ injuries that was obtained in the immediate postoperative period. *p<0.05 between both groups according to Pearson chi-square test, revealing a more favorable evolution for TL group patients, " data was not available for all cases at initial presentation, "available cases for two-sided analysis. TL: translabyrinthine, RS: retrosigmoid, $\mathrm{CN}$ : cranial nerve 
postoperative period and the one-year follow-up $(p<0.001)$. However, RS group was associated with a higher rate of facial paresis than TL group for all three periods $(p<0.05)$ and the difference remained constant $(p=0.016)$. Thus, the evolution of proportion was not significantly influenced by the surgical approach ( $p=0.942)$ (Fig. 1).

\section{Vestibular complications}

Two-sided analysis was performed in all cases for which pre- and postoperative data were available. Results revealed a higher rate of ataxia in patients surgically treated by RS approach $(p=0.019)$ (Table 2). Incidence of vertigo was not associated with any of the two surgical approaches $(p=0.886)$ (Table 2).

\section{Headache}

Regarding the incidence of headache, no difference was found between both groups. Development postoperatively did not correlate with the surgical approach $(p=0.466)$ (Table 2).

\section{Tinnitus}

Of the available cases for two-sided analysis, postoperative tinnitus development was greater in patients treated by RS surgical approach ( $p=0.039)$ (Table 2$)$.

\section{Cranial nerves injuries}

In the preoperative period, the RS group revealed a higher rate of cranial nerve injuries. In their own respective groups, six patients complained of hypoesthesia ipsilaterally to the lesion and one presented with dysphagia. Postoperatively, higher incidence of cranial nerve injuries was correlated with the RS approach $(p=0.016)$ (Table 2).

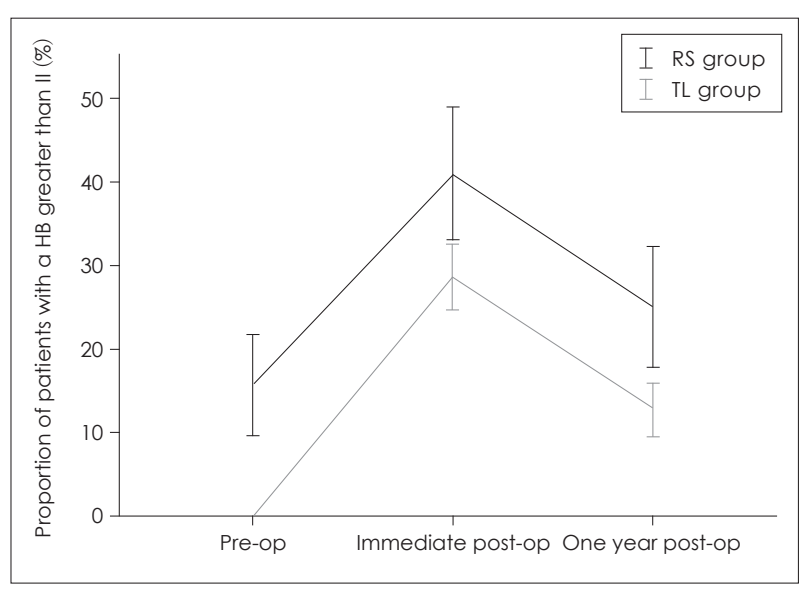

Fig. 1. Evolution in time of facial function in relation to the surgical approach. TL: Translabyrinthine, RS: Retrosigmoid, HB: HouseBrackmann.

\section{Vascular complications}

In TL group, 2 cases were complicated with cerebellar hematomas, 1 case with pontine ischemia and 2 cases with both cerebellar and pontine ischemia, whereas in RS group, cerebellar hematomas were recorded in 3 cases. Overall, no difference was noted between the two groups (Table 3).

\section{CSF leak and meningitis}

CSF leaks were diagnosed by direct visualization or CSF analysis for $\beta 2$ transferrin. All cases of meningitis were diagnosed through standard CSF culture.

In TL group, a total of 10 patients had CSF leaks (7.8\%); 8 patients exhibited wound leakage and 2 had rhinorrhea. Of those 8 patients with wound leakage, 3 were treated with lumbar drains and 2 with dressings and bed rest. The last 3 patients required a surgical treatment; one of those was complicated with meningitis. In addition, rhinorrhea was recorded in two patients, ultimately necessitating a surgical procedure. One of those 2 patients subsequently developed meningitis.

In RS group, CSF leak was observed in 5 patients $(12.8 \%)$. CSF leakage through surgical wounds was seen in 2 patients, both treated with lumbar drains. Rhinorrhea was observed in 2 cases, one treated with lumbar drain and one treated surgically with concomitant meningitis. One case exhibited features of both wound leakage and rhinorrhea and was treated by surgical repair. Meningitis was also recorded in that patient.

The difference in CSF leak between both groups was found to be non-significant $(p=0.331)$. Regarding the total rate of meningitis, a non-statistically significant difference was found $(p=0.902)$ (Table 3).

\section{Hospital stay}

The length of stay was measured from the day of surgery to discharge. The difference between both groups was significant. Mean hospital stay was 7.47 days for TL group and 15.97 days for RS group $(p<0.05)$ (Table 3$)$. No mortalities were observed per-hospitalization.

Table 3. Postoperative parameters and surgical complications

\begin{tabular}{lcc}
\hline \multicolumn{1}{c}{ Post-op parameters/ } & \multicolumn{2}{c}{ Approach } \\
\cline { 2 - 3 } complications & TL group & RS group \\
\hline Total number of cases & 129 & 39 \\
Meningitis, $\mathrm{n}(\%)$ & $6(4.74)$ & $2(5.1)$ \\
CSF leak, $\mathrm{n}(\%)$ & $10(7.8)$ & $5(12.8)$ \\
Vascular complications, $\mathrm{n}(\%)$ & $5(3.9)$ & $3(7.7)$ \\
Mean hospital stay (days) & $7.47^{*}$ & $15.97^{*}$ \\
\hline
\end{tabular}

Vascular complications include cerebellar/cerebello-pontine angle hematoma, cerebellar ischemia and pontine ischemia. * $p<0.05$ according to student t-test. TL: translabyrinthine, RS: retrosigmoid, CSF: cerebrospinal fluid 


\section{Discussion}

The present study was designed to compare the morbidity rate between the RS and TL approach for the treatment of VS. Our analysis revealed that both groups had tumors of similar estimated surface, extension in and anterior to the longitudinal axis of the IAC as well as a comparable degree of resection. Thus, the two groups were considered comparable and subsequent analysis of morbidity was performed. Since in our center, sigmoid sinus was never an obstacle for a TL, neither for a RS approach, we never noted its position.

The potentially wide symptomatic spectrum described by patients with VS is well represented in patients with large lesions. Clinical presentation of patients with large VS involves hearing loss $(96-100 \%)$, tinnitus ( $42-46 \%)$, trigeminal dysfunction $(10-14 \%)$ and the compressive effect on the middle cerebellar peduncle and cerebellum (44-88\%) [7]. In previous studies, the largest diameter has been the measurement of choice for tumor size $[12,16,20,21]$. Considering the anatomical location of the surrounding structures and the variability of tumor shape, we believe that the estimated tumor surface measured by multiplying the two largest diameters on axial plane is a more optimal method for tumor size measurement and a better representation of its morphology. In fact, the longitudinal diameter reflects the anterior extension and the potential facial nerve compression on the adjacent petrous bone whereas the transverse diameter contributes to the structural damage by compressing the cerebellum as well as the vestibulocochlear and facial nerve near the IAC. In addition, the bi-axial tumor size is more representative of the efficacy and facility of resection: better exposure and shorter dissection are not only related to the largest diameter but rather to the tumor's dimensions.

Facial nerve function has been attributed to be the best indicator of quality of life [22]. In addition to complete tumor removal [11], its preservation is described as a main objective in VS resection [16]. Choice of surgical approach for the treatment of VS remains controversial, and whether the TL or the RS approach is associated with a lower risk of facial nerve injury is unclear. Studies have revealed that facial function is associated with a higher risk of immediate postoperative paresis following RS approach [12]. Although tumor size is considered a poor predictor of facial nerve preservation $[20,23]$, the RS approach seems to have an independent harmful effect on facial nerve injury, as depicted in studies where comparable tumor size were analyzed [12]. On the other hand, Ansari, et al. noted that the RS approach seemed more beneficial in facial nerve maintenance for tumors $1.5-3 \mathrm{~cm}$ [1].

Although tumor size and degree of resection affect the rate of facial paresis [8], similar measurements for these parameters were observed in both groups enabling comparison. Despite neurophysiological facial nerve monitoring, facial dysfunction has been observed in a fair proportion of patients in both groups. Such paresis was mainly observed in cases of highly adherent tumors and resulted from traction injury. Association between the TL approach and a lower risk of facial paresis due to better localization of the facial nerve [16] was not confirmed in our study. Both approaches revealed a similar rate of facial nerve dysfunction. These findings are supported by recent reports in the literature [6,16]. Indeed, Guergel, et al. [12] observed in a systematic review that the surgical approach did not seem to influence facial nerve outcome.

According to Ansari, et al. [1], the surgical approach does not influence the rate of non-facial cranial nerve injuries. Our results do not correspond to those findings, revealing a higher rate of cranial nerve injuries in patients treated by RS approach. This is not surprising, since a wider exposure is achievable through the TL approach [24], allowing meticulous nerve root dissection.

CSF leak is the most common complication following VS surgical resection, with an incidence reaching up to $30 \%$ of cases [25]. Incisional leaks result from an abnormal communication with the subarachnoid space [26], while rhinorrhea is a consequence of an iatrogenic communication with the middle ear and subsequent access to the Eustachian tube through pneumatized air cell tracts. Thus, optimal wound closure and adequate obliteration of air cells with bone wax are necessary steps in preventing incisional and rhinorrheal CSF leaks [16,27]. Few authors, including Ansari, et al., revealed a higher rate of CSF leak in patients that underwent a TL surgical resection rather than RS $[9,23]$. Despite an incidence of CSF leak similar to the literature $[9,26]$, our results did not illustrate any difference of CSF leak between both groups. Supporting evidence reveled a comparable rate of CSF leak for both groups $[26,28,29]$. We recommend, in addition to proper closure and obliteration of air cells, abdominal fat placement of the drilled-out region following TL tumor resection. In our experience, these key steps lower the incidence of leakage [25]. Additional precautions such as Eustachian tube closure with aponeurosis patches through the epitympanum (Glasscock's technique) have been used in some centers $[7,16]$ for diminishing the risk of CSF leak following TL approach. For all patients in this study, postoperative pressure dressings were applied and anti-Valsalva instructions were explained.

Meningitis is a serious complication of VS surgery. Its rate often correlates with CSF leak incidence [26]. Similarly to CSF leak analysis, our study revealed no difference in the rate of meningitis between both groups, suggesting that such 
infection often results from CSF fistula. Patients diagnosed with meningitis by CSF cell count were initially started on empiric intravenous antibiotics. Following CSF culture results, antibiotics were narrowed according to sensitivity and continued for a total of 2 weeks.

Vascular complications following VS resection have been described in up to $7 \%$ of patients [20]. These include arterial, venous infarcts and hematomas from various locations around the surgical site. Cerebellopontine angle hematomas usually have a poor prognosis, with a mortality rate reaching $50 \%$ in some series [30]. Ischemic complications, especially pontine infarcts, also have a major postoperative impact on overall morbidity. Particular attention in arachnoid plane preservation is a key element to the dissection. It allows preservation of the recurrent perforating and the distal anterior inferior cerebellar artery [31] and prevents ischemic consequences. Our study, for which House, et al. [8] reproduced the results, illustrated a similar rate of overall vascular complications in both treatment groups. Thus, the belief that cerebellar retraction might lead to higher rate of infarcts [32] was not observed in our study. This suggests that precise surgical technique rather than the choice of approach is the major factor in preventing vascular complications.

Evaluation of postoperative vertigo and ataxic gait was performed one month following surgical resection. Those symptoms are often attributed to the surgical act, but rarely considered true complications [16]. It is not uncommon that in the postoperative period, patients exhibit disequilibrium and vertigo [24,27]. The extent of symptoms depends on the preoperative vestibular function. As the tumor grows, the vestibular nerve becomes damaged. However, central compensation seems to occur in patients with slow growing tumors allowing for symptomatic stabilization. If partial peripheral function remains despite tumor enlargement, acute vertigo and ataxic gait might be observed following resection and often result from surgical transection of one branch of the vestibular nerve [33]. However, our experience suggests that profound preoperative vestibular deficit is associated with mild to absent ataxia and vertigo postoperatively. For that reason, in order to predict the postoperative symptomatic course, we recommend preoperative videonystagmography for all patients. Ho, et al. [15] compared the TL and RS approaches and concluded that both approaches had similar incidence of vestibular symptoms including vertigo and ataxia. The present study illustrates a higher rate of ataxic gait in patients treated by RS approach. Based on the literature, we believe that the need for cerebellar retraction in the RS approach plays a major role in the development of disequilibrium by direct compression of the cerebellum [34]. This complaint does not seem transient considering its persistence one month postoperatively. Supporting evidence came from Kane, et al. who recorded that up to $65 \%$ of patients complain of dizziness and unsteadiness 3 months to 7 years after RS approach [21]. Regardless of the chosen surgical approach, we recommend vestibular rehabilitation following surgery for all patients with new-onset symptoms of disequilibrium or vertigo.

Tinnitus is another common symptom in patients with VS $(60-80 \%)$ [35] which often affects quality of life. In addition to its manifestation preoperatively, it often presents in patients following VS surgery [27]. Our analysis revealed a statistically significant higher rate of new-onset tinnitus in patients treated by RS approach. Studies have shown that the TL method might be associated with a greater incidence of new-onset tinnitus because of the loss of peripheral excitations [35]. Nevertheless, our findings are supported by a study performed by Harcourt, et al. who noticed that sectioning the cochlear nerve might lead to symptomatic improvement [13].

The incidence of postoperative headaches can reach up to $65 \%$ of cases in patients operated for VS [24]. Despite optimal surgical technique, headaches more often result from the RS rather than the TL approach. Several causes have been identified including dural adhesion to nuchal muscles with subsequent pain upon neck movements, direct dural closure leading to increased tension, cerebellar retraction, occipital nerve entrapment or sectioning during scalp dissection and injury to nuchal muscles $[9,24,27,36,37]$. On the opposite, a recent study showed no statistical difference in the rate of newonset headache between the two approaches [9]. Our results lead to a similar conclusion: a comparable incidence of postoperative headache was recorded.

Short postoperative hospital stay is an important preventive measure to nosocomial complications. Our study revealed a shorter hospital stay in patients treated by TL rather than RS approach. It is not surprising since, according to our analysis, the overall complication rate was greater in patients that underwent RS VS surgery. We believe that postoperative ataxic gait is the main determinant of a longer hospital stay, considering the lengthy rehabilitation prior to discharge.

The main limitation of our study is its retrospective aspect. Hence, for some parameters, data was not available, decreasing the power of the analysis. In addition, a possible followup bias could have occurred. However, considering a mean follow-up period of 17 months in the TL group and 45 months in the RS group, adequate analysis of the required parameters up to one year was possible for most cases. An additional limitation is the relatively small number of cases in the RS group compared to the TL group. Moreover, a confounding bias 
likely occurred: patients from the TL group were operated by a different surgeon than RS group patients. Finally, the surgeon's preference of surgical approach in each respective group constitutes a selection bias.

Despite these limitations, our analysis reveals that the TL approach is associated with a lower risk of surgical complications than the RS approach. Previous studies comparing both approaches have been done, but no consensus for the choice of surgical approach has been agreed on. For that reason, we believe that these analyses were necessary to provide additional evidence for surgical treatment of VS.

In conclusion, for VS in which hearing preservation is not considered, TL surgical approach is preferred. According to our analysis, it is associated with a lower risk of cranial nerve injuries, ataxic gait and tinnitus and leads to a shorter hospital stay. The lower morbidity rate associated with the TL approach dictates surgical planning and allows improvement in quality of life.

\section{Conflicts of interest}

The authors have no financial conflicts of interest.

\section{REFERENCES}

1) Ansari SF, Terry C, Cohen-Gadol AA. Surgery for vestibular schwannomas: a systematic review of complications by approach. Neurosurg Focus 2012;33:E14.

2) Brennan JW, Rowed DW, Nedzelski JM, Chen JM. Cerebrospinal fluid leak after acoustic neuroma surgery: influence of tumor size and surgical approach on incidence and response to treatment. J Neurosurg 2001;94:217-23.

3) Briggs RJ, Luxford WM, Atkins JS Jr, Hitselberger WE. Translabyrinthine removal of large acoustic neuromas. Neurosurgery 1994;34: 785-90.

4) Charabi S, Tos M, Thomsen J, Charabi B, Mantoni M. Vestibular schwannoma growth--long-term results. Acta Otolaryngol Suppl 2000; 543:7-10.

5) Charalampakis S, Koutsimpelas D, Gouveris H, Mann W. Post-operative complications after removal of sporadic vestibular schwannoma via retrosigmoid-suboccipital approach: current diagnosis and management. Eur Arch Otorhinolaryngol 2011;268:653-60.

6) Cheng S, Naidoo Y, da Cruz M, Dexter M. Quality of life in postoperative vestibular schwannoma patients. Laryngoscope 2009;119: 2252-7.

7) Dandy WE. An operation for the total removal of cerebellopontine (acoustic) tumors. Surg Gynecol Obstet 1925;41:129-48

8) House JW, Brackmann DE. Facial nerve grading system. Otolaryngol Head Neck Surg 1985;93:146-7.

9) Darrouzet V, Martel J, Enée V, Bébéar JP, Guérin J. Vestibular schwannoma surgery outcomes: our multidisciplinary experience in 400 cases over 17 years. Laryngoscope 2004;114:681-8.

10) Elhammady MS, Telischi FF, Morcos JJ. Retrosigmoid approach: indications, techniques, and results. Otolaryngol Clin North Am 2012; 45:375-97.

11) Fishman AJ, Marrinan MS, Golfinos JG, Cohen NL, Roland JT Jr. Prevention and management of cerebrospinal fluid leak following vestibular schwannoma surgery. Laryngoscope 2004;114:501-5.

12) Gurgel RK, Dogru S, Amdur RL, Monfared A. Facial nerve outcomes after surgery for large vestibular schwannomas: do surgical approach and extent of resection matter? Neurosurg Focus 2012;33: E16.

13) Harcourt J, Thomsen J, Tos M. Translabyrinthine vestibular schwannoma surgery: postoperative tinnitus and cochlear nerve integrity. Auris Nasus Larynx 1997;24:21-6.

14) Hegarty JL, Jackler RK, Rigby PL, Pitts LH, Cheung SW. Distal anterior inferior cerebellar artery syndrome after acoustic neuroma surgery. Otol Neurotol 2002;23:560-71.

15) Ho SY, Hudgens S, Wiet RJ. Comparison of postoperative facial nerve outcomes between translabyrinthine and retrosigmoid approaches in matched-pair patients. Laryngoscope 2003;113:2014-20.

16) Hoffman RA. Cerebrospinal fluid leak following acoustic neuroma removal. Laryngoscope 1994;104(1 Pt 1):40-58.

17) Day JD, Chen DA, Arriaga M. Translabyrinthine approach for acoustic neuroma. Neurosurgery 2004;54:391-5.

18) House WF. Transtemporal bone microsurgical removal of acoustic neuromas. Evolution of transtemporal bone removal of acoustic tumors. Arch Otolaryngol 1964;80:731-42.

19) Jacob A, Robinson LL Jr, Bortman JS, Yu L, Dodson EE, Welling DB. Nerve of origin, tumor size, hearing preservation, and facial nerve outcomes in 359 vestibular schwannoma resections at a tertiary care academic center. Laryngoscope 2007;117:2087-92.

20) Jung S, Kang SS, Kim TS, Kim HJ, Jeong SK, Kim SC, et al. Current surgical results of retrosigmoid approach in extralarge vestibular schwannomas. Surg Neurol 2000;53:370-7.

21) Kane NM, Kazanas S, Maw AR, Coakham HB, Torrens MJ, Morgan $\mathrm{MH}$, et al. Functional outcome in patients after excision of extracanalicular acoustic neuromas using the suboccipital approach. Ann R Coll Surg Engl 1995;77:210-6.

22) Lalwani AK, Butt FY, Jackler RK, Pitts LH, Yingling CD. Facial nerve outcome after acoustic neuroma surgery: a study from the era of cranial nerve monitoring. Otolaryngol Head Neck Surg 1994;111: 561-70.

23) Lunsford LD, Niranjan A, Flickinger JC, Maitz A, Kondziolka D. Radiosurgery of vestibular schwannomas: summary of experience in 829 cases. J Neurosurg 2005;102 Suppl:195-9.

24) Mamikoglu B, Esquivel CR, Wiet RJ. Comparison of facial nerve function results after translabyrinthine and retrosigmoid approach in medium-sized tumors. Arch Otolaryngol Head Neck Surg 2003;129: 429-31.

25) Maurer J, Frommeld T, Mann W. Vestibular function after acoustic neuroma removal with preservation of one branch of the vestibular nerve. Otol Neurotol 2002;23:749-54.

26) Misra BK, Purandare HR, Ved RS, Bagdia AA, Mare PB. Current treatment strategy in the management of vestibular schwannoma. Neurol India 2009;57:257-63.

27) Saliba I, Shinghal T, Nehme J, Dufour JJ. Eustachian tube obliteration in translabyrinthine vestibular schwannoma excision: cerebrospinal fluid rhinorrhea and middle ear status. J Otolaryngol Head Neck Surg 2011;40:367-75.

28) Samii M, Matthies C. Management of 1000 vestibular schwannomas (acoustic neuromas): hearing function in 1000 tumor resections. Neurosurgery 1997;40:248-60.

29) Schaller B, Baumann A. Headache after removal of vestibular schwannoma via the retrosigmoid approach: a long-term follow-upstudy. Otolaryngol Head Neck Surg 2003;128:387-95.

30) Staecker H, Nadol JB Jr, Ojeman R, Ronner S, McKenna MJ. Hearing preservation in acoustic neuroma surgery: middle fossa versus retrosigmoid approach. Am J Otol 2000;21:399-404.

31) Sughrue ME, Yang I, Rutkowski MJ, Aranda D, Parsa AT. Preservation of facial nerve function after resection of vestibular schwannoma. Br J Neurosurg 2010;24:666-71.

32) Suh JH, Barnett GH, Sohn JW, Kupelian PA, Cohen BH. Results of linear accelerator-based stereotactic radiosurgery for recurrent and newly diagnosed acoustic neuromas. Int J Cancer 2000;90:145-51.

33) Lanman TH, Brackmann DE, Hitselberger WE, Subin B. Report of 
190 consecutive cases of large acoustic tumors (vestibular schwannoma) removed via the translabyrinthine approach. J Neurosurg 1999; 90:617-23

34) Tos M, Thomsen J, Harmsen A. Results of translabyrinthine removal of 300 acoustic neuromas related to tumour size. Acta Otolaryngol Suppl 1988;452:38-51.

35) Wiet RJ, Kazan RP, Raslan W, Herzon GD. Complications in the ap- proach to acoustic tumor surgery. Ann Otol Rhinol Laryngol 1986; 95(1 Pt 1):28-31.

36) Wiet RJ, Mamikoglu B, Odom L, Hoistad DL. Long-term results of the first 500 cases of acoustic neuroma surgery. Otolaryngol Head Neck Surg 2001;124:645-51.

37) Yasargil MG, Fox JL. The microsurgical approach to acoustic neurinomas. Surg Neurol 1974;2:393-8. 